Revista Latinoamericana de la Papa 23 (1): 76 - 85, 2019

ISSN: 1853-4961

http://ojs.papaslatinas.org/index.php/rev-alap/index

\title{
Selección de genotipos de papa (Solanum tuberosum L.) adaptados a las condiciones agroecológicas del Estado Mérida, Venezuela
}

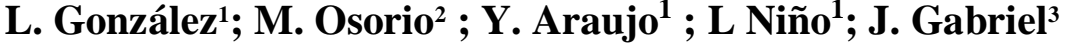

Recibido: $22 / 05 / 2018$

Aceptado: 20/06/2019

Accesible en línea: Junio de 2019

\section{Resumen}

La papa es considerada uno de los principales cultivos agrícolas en el mundo en cuanto a superficie. En Venezuela, ocupa el octavo lugar en producción y primer lugar de las raíces y tubérculos. Para consumo fresco predomina el cultivar Granola, de origen alemán, susceptible a candelilla tardía (Phytophthora infestans). En este contexto, el objetivo de la presente investigación fue seleccionar los genotipos de papa (Solanum tuberosum L.) adaptados a las condiciones agroecológicas del estado Mérida. La emergencia de los genotipos estuvo por encima del $80 \%$ en todas las localidades consideradas. Los valores de AUDPC variaron de 0 hasta 7865 en el testigo susceptible. Siete clones y cinco cultivares no presentaron la enfermedad, mientras los mayores valores de AUDPC fueron para los cultivares Granola, Diacol Capiro, Andinita y Montañita, por lo que estos materiales mostraron baja resistencia a candelilla tardía. El rendimiento promedio más alto se registró en el clon 301002.6 (49.63 t.ha ${ }^{-1}$ ), mientras que el menor se obtuvo en Única - Peruana (4.14 t.ha ${ }^{-1}$ ). El $85 \%$ de los materiales superaron al testigo en rendimiento. De acuerdo a la prueba múltiple de Duncan se formaron 23 grupos, observándose que estadísticamente existen diferencias altamente significativas entre los genotipos evaluados $(\mathrm{p}<0,001)$. La materia seca (\% sólidos) estuvo por encima del $14 \%$, no obstante, superaron el $15 \%$ de defectos totales por lo que solo resultaron aptas para la industrias los clones 382121.25, 382151.22 y los cultivares María Bonita, Iniafrit, Fripapa y Diacol Capiro.

Palabras claves adicionales: procesamiento, caracterización, candelilla, raíces y tubérculos.

\section{Selection of potato genotypes (Solanum tuberosum L.) adapted to the agroecological conditions of the State of Mérida, Venezuela}

\section{Summary}

The potato is considered one of the main agricultural crops in the world in terms of area. In Venezuela, it occupies the eighth place in production and first place of the roots and tubers. For fresh consumption the cultivar Granola, of German origin, susceptible to late blight (Phytophthora infestans) predominates. In this context, the objective of this research was to select genotypes adapted to the agroecological conditions of the State of Mérida. The

Correo de contacto. E-mail: lcgonzalez@inia.gob.ve., oasisuniversal@gmail.com Centro de Investigaciones Agrícolas del Estado Mérida (CIAE-Mérida). CP 4525. Mérida, Venezuela Centro Nacional de Investigaciones Agropecuarias. INIA-CENIAP. CP 2105.3, Maracay, Venezuela. Universidad Estatal del Sur de Manabí (UNESUM), Jipijapa, Manabí, Ecuador. E-Mail: julio.gabriel@unesum.edu.ec 
emergence of the genotypes was above $80 \%$ in all the localities considered. The AUDPC values varied from 0 to 7865 in the susceptible control. Seven clones and five cultivars did not present the disease, while the highest values of AUDPC were for the cultivars Granola, Diacol Capiro, Andinita and Montañita, for which these materials showed low resistance to late blight. The highest average yield was registered in clone 301002.6 (49.63 t.ha ${ }^{-1}$ ), while the lowest was obtained in Única Peruana $\left(4.14 \mathrm{t}^{\mathrm{h}} \mathrm{ha}^{-1}\right) .85 \%$ of the materials outperformed the witness in performance. According to Duncan's multiple test, 23 groups were formed, observing that statistically there are highly significant differences between the genotypes evaluated $(\mathrm{p}<0.001)$. The dry matter (\% solids) was above $14 \%$, however, they exceeded $15 \%$ of total defects, so only the clones 382121.25 , 382151.22 and the cultivars María Bonita, Iniafrit, Fripapa and Diacol Capiro were suitable for the industry.

Additional keywords: processing, characterization, late blight, roots and tubers.

\section{Introducción}

La papa (Solanum tuberosum L.) es considerada como uno de los principales cultivos agrícolas en el mundo en cuanto a superficie. Representa junto con el maíz, trigo y arroz los cuatro rubros alimenticios básicos de la seguridad y soberanía alimentaria (Madroñero et. al., 2013). Está adaptada a una amplia gama de climas y se encuentra tanto en ambientes tropicales como templados y a altitudes desde el nivel del mar hasta los 4000 metros (Poehlman, 2003). En Venezuela, ocupa el octavo lugar en producción y el primer lugar de las raíces y tubérculos (Niño et al., 2004). Los principales estados productores son Mérida, Trujillo, Táchira, Lara, Aragua y Carabobo. La producción de papa en el país se destina tanto al consumo fresco como para la industria. Para el consumo fresco predomina el cultivar Granola, de origen alemán y susceptible a candelilla tardía (Phytophthora infestans). Además, se cultivan otros cultivares de origen colombiano (Rodríguez et al., 2009), así como de otros países y en menor proporción, los cultivares nacionales. Los agricultores demandan cultivares de ciclo corto, de tubérculos blancos y con buenas características para el manejo en postcosecha. En este contexto, el objetivo del presente trabajo fue seleccionar los genotipos de papa (Solanum tuberosum L.) adaptados a las condiciones agroecológicas del estado Mérida.

\section{Materiales y métodos}

Se evaluaron clones promisorios, progenies, cultivares comerciales y locales. Los ensayos se realizaron en 11 localidades del estado Mérida, Venezuela durante cinco años (2006-2010). Bajo un diseño experimental en bloques completamente aleatorios con 10 tratamientos y cuatro repeticiones. Los experimentos fueron analizados en series de experimentos (Gabriel et al., 2017).

El comportamiento de los genotipos se determinó evaluando la emergencia, respuesta a candelilla tardía (Phytophthora infestans) rendimiento comercial y aptitud para fritura. Además se realizó una caracterización morfológica.

Emergencia: La emergencia se realizó a los 45 días después de la siembra, realizando contajes en cada hilo y estimando el promedio de la parcela experimental.

Respuesta a candelilla tardía (Phytophthora infestans)

La severidad de la enfermedad se evalúa como el porcentaje de área foliar 
infectada. Esta variable se registra a lo largo del desarrollo del ensayo junto con la fecha de cada lectura. Los datos son recolectados en cada parcela o unidad experimental (cada clon o cultivar dentro de cada repetición), se pueden registrar manualmente o con dispositivos electrónicos con la finalidad de reducir el tiempo y el costo de la recolección de datos y su análisis posterior (Forbes et al., 2014)

Para evaluar el daño causado por la candelilla tardía ( $P$. infestans) se estimó visualmente el área de la planta afectada con respecto al total de la parcela, cada siete días, a partir de los 42 días después de la siembra. Se realizaron evaluaciones de los genotipos por reacción a esta enfermedad utilizando la escala visual recomendada por Bonierbale et al., (2008), con la cual se calculó el área bajo la curva del progreso de la enfermedad (AUDPC)

Rendimiento: Para determinar el rendimiento a la cosecha se evaluaron los dos hilos centrales de cada parcela y se calculó el rendimiento comercial (t.ha $\left.{ }^{-1}\right)$. Los datos fueron sometidos a análisis de varianza con el programa estadístico SAS University. La comparación de medias se llevó a cabo mediante la Prueba de Duncan (Gabriel et al., 2017).

Aptitud para fritura: Las pruebas de fritura fueron realizadas por la empresa PEPSICO ALIMENTOS S.C.A., Planta La Grita, en el estado Táchira, con muestras de $10 \mathrm{~kg}$ de papa por cada genotipo procedente de los ensayos. $\mathrm{La}$ empresa asignó la clasificación de apta o no apta para la agroindustria considerando las características de defectos totales (suma de color indeseable, \% de verdeo y decoloración interna y externa de las hojuelas durante el proceso de freído).
Caracterización morfológica: Las variables morfológicas se evaluaron utilizando los descriptores del Centro Internacional de la Papa (CIP) (Huamán y Gómez, 1994).

\section{Resultados y discusión}

\section{Emergencia}

Los resultados mostraron que la emergencia de los genotipos estuvo por encima del $80 \%$ en promedio para todas las localidades consideradas. Solo 14 genotipos de los 35 evaluados no superaron al testigo, el cual presento $94,12 \%$ (Granola), el mayor porcentaje de emergencia fue para el clon 382151.22 (99.5\%), mientras que Fripapa INIA presentó el menor valor $65.94 \%$. Estos resultados coinciden con los obtenidos por Meza et al. (2009), quienes evaluaron clones de papa en la localidad de Cuencas, estado Trujillo. No obstante, en el trabajo realizado por Meza et al. (2010) en Marajabú Estado Trujillo se observaron valores de emergencia desde 40 a $92 \%$, para 16 clones evaluados, estos resultados difieren de los obtenidos en la presente investigación probablemente debido a las condiciones de desarrollo y manejo agronómico de ambos ensayos.

\section{Incidencia candelilla}

Los valores de AUDPC variaron de 0 hasta 7865 en el testigo susceptible. Siete clones y cinco cultivares no presentaron la enfermedad (0), mientras los mayores valores de AUDPC fueron para los cultivares Granola (7865), Diacol Capiro (5747.5), Andinita (4232,5) y Montañita (2039.88), por lo que estos materiales mostraron baja resistencia a candelilla tardía. Lo cual coincide con los resultados obtenidos en evaluaciones donde se han usado los cultivares señalados y se notó baja resistencia a candelilla tardía, lo cual 
indica que son genotipos con baja resistencia y/o tolerancia a candelilla tardía (González et al., 2011). En este grupo de genotipos evaluados se encuentran aquellos que fueron generados por el CIP de la Población A (resistencia vertical) y Población B (resistencia horizontal en ausencia de genes R) por lo cual los niveles de resistencia son variables. Estos resultados son similares en cuanto a la variabilidad en la respuesta a candelilla tardía a los obtenidos por Rodríguez et al. (2008), que evaluaron 190 clones de la población B procedentes del CIP por resistencia a candelilla y rendimiento.

\section{Rendimiento}

El rendimiento promedio más alto de las localidades (Tabla 1), se registró en el clon 301002.6 (49.63 t.ha $\left.{ }^{-1}\right)$, mientras que el menor se obtuvo en el cultivar Única Peruana $\left(4,14\right.$ t.ha $\left.^{-1}\right)$. El $85 \%$ de los materiales superaron al testigo en rendimiento. No obstante de acuerdo a la prueba de medias de Duncan se formaron 23 grupos, observándose que estadísticamente existen diferencias significativas entre los genotipos evaluados $(\mathrm{p}<0.001)$.

Tabla 1. Rendimiento promedio $\left(\mathrm{t} . \mathrm{ha}^{-1}\right)$ de Clones promisorios, progenies y cultivares de papa evaluados en localidades del Estado de Mérida, Venezuela.

\begin{tabular}{lcl}
\hline Genotipos & $\begin{array}{c}\text { Rendimiento promedio } \\
\left(\text { t.ha }{ }^{-1}\right)\end{array}$ & Grupos \\
\hline 301002.6 & 49.6 & A \\
391580.30 & 46.8 & AB \\
391011.17 & 43,9 & ABC \\
393258.44 & 42,4 & ABCD \\
393371.159 & 40,0 & BCDE \\
Andinita & 39,8 & BCDE \\
393280.82 & 39,7 & BCDE \\
392633.54 & 38,4 & BCDEF \\
382121.25 & 36.6 & CDEFG \\
393349.68 & 33.8 & DEFGH \\
386528.7 & 33.3 & DEFGH \\
392639.1 & 32.5 & EFGHI \\
393658.44 & 29.8 & FGHIJ \\
393194.27 & 29.2 & FGHIJ \\
Achiranax TS-4 (990021) & 29.2 & FGHIJ \\
382151.22 & 29.0 & FGHIJ \\
AchiranaxTPS-67 (96003) & 27.4 & GHIJK \\
393085.5 & 26.6 & GHIJK \\
TPS-25XTPS-67 IP88008) & 25.3 & HIJKL \\
MF-IIxTPS-67 (IP88004) & 25.2 & HIJKL \\
TPS-25 XTPS-13 (IP88007) & 24.7 & HIJKLM \\
\hline
\end{tabular}




\begin{tabular}{lll}
\hline 393194.1 & 24.7 & HIJKLM \\
Tibisay & 23.1 & IJKLMN \\
393371.58 & 21.8 & JKLMNO \\
SN Táchira & 20.1 & JKLMNOP \\
Maria Bonita & 18.3 & KLMNOPQ \\
Montañita & 17.7 & KLMNOPQ \\
Cartayita & 16.3 & LMNOPQ \\
393280.57 & 14.9 & MNOPQ \\
Esperanza & 13.5 & NOPQR \\
Iniafrit & 13.0 & NOPQR \\
Granola (testigo) & 11.8 & PQR \\
Carabay & 11.4 & PQR \\
Fripapa INIA & 10.6 & PQR \\
Diacol Capiro & 8.8 & QR \\
Unica Peruana & 4.1 & $\mathrm{R}$ \\
\hline
\end{tabular}

La respuesta de clones y cultivares de papa en relación al rendimiento evaluado en TM fueron diferentes dentro y entre localidades, lo que confirma que este componente es una característica varietal y depende de la interacción genotipo-ambiente (Monar et al., 2015). Esta tendencia se observó en trabajos de investigación donde se evaluaron genotipos en diferentes localidades (Meza et al., 2009; Martínez et al., 2005; Rodríguez et al., 2008).

\section{Aptitud para fritura}

Se observó en los genotipos evaluados, que la materia seca (\% sólidos ) se encuentra por encima del $14 \%$ como lo exige la empresa procesadora, no obstante, al obtener lo defectos totales (suma de color indeseable, \% de verdeo y decoloración interna y externa de las hojuelas durante el proceso de freído) los mismos superan el 15\% de defectos totales en un $80 \%$ de las muestras analizadas, por lo tanto estas no son aptas para el procesamiento industrial (Tabla 2), bajo las condiciones de manejo agronómicos usadas en estos experimentos, así como la altura donde se llevaron a cabo los ensayos. El manejo poscosecha también afecta el procesamiento.

Aptas para la industria resultaron los siguientes genotipos: Los clones 382121.25, 382151.22 y los cultivares María Bonita, Iniafrit, Fripapa y como testigo industrial: Diacol Capiro. Evaluaciones de cultivares y clones de papa realizadas en seis localidades del estado Mérida, los cultivares Iniafrit, Fripapa INIA y Diacol Capiro fueron aptas para la industria y el cultivar Tibisay no apta, lo cual coincide con los resultados obtenidos en la presente investigación (Niño et al, 2004). 
Tabla 2. Valores Promedio del Análisis realizado a muestras de papa para procesamiento industrial por Empresa procesadora de Snacks América Latina.

\begin{tabular}{|c|c|c|c|c|c|c|}
\hline Genotipos & $\begin{array}{l}\text { Materia } \\
\text { seca } \\
\text { (Rangos) }\end{array}$ & $\begin{array}{l}\text { \% de color } \\
\text { indeseable }\end{array}$ & $\begin{array}{c}\% \text { de } \\
\text { decoloración } \\
\text { interna } \\
\text { hojuelas } \\
\text { (rangos) }\end{array}$ & $\begin{array}{c}\% \text { de } \\
\text { decoloración } \\
\text { externa } \\
\text { hojuelas } \\
\text { (rangos) }\end{array}$ & $\begin{array}{c}\% \\
\text { verde }\end{array}$ & Calificación \\
\hline Diacol Capiro & 15.5 & 3.76 & 4.03 & 6.18 & 1.22 & Apta \\
\hline Maria Bonita & 18.2 & 0.82 & 1.8 & 2.63 & 0.1 & Apta \\
\hline Fripapa INIA & 18.2 & 2.20 & 7.14 & 8.25 & 3.38 & Apta \\
\hline Iniafrit & 17.025 & 4.24 & 7.14 & 6.91 & 2.53 & Apta \\
\hline 382151.22 & 16.5 & 4.89 & 1.8 & 5.78 & 3.18 & Apta \\
\hline 382121.25 & 15.2 & 3.19 & 2.8 & 4.12 & 3.23 & Apta \\
\hline TPS-25 XTPS-13 (IP88007) & 15.4 & 5.9 & 4.2 & 1.18 & 1.7 & Apta \\
\hline 301002.6 & 14.6 & 100 & 0 & 0 & 0 & No apto \\
\hline 386528.7 & 14 & 15.62 & 3.79 & 11.8 & 2.90 & No apto \\
\hline 391011.17 & 14.9 & 100 & 0 & 0 & 0 & No apto \\
\hline 391580.30 & 16.8 & 95.49 & 0 & 0 & 0 & No apto \\
\hline 392633.54 & 15.3 & 100 & 0 & 0 & 0 & No apto \\
\hline 392639.1 & 17.7 & 100 & 0 & 0 & 0 & No apto \\
\hline 393085.5 & 16 & 53.79 & 6.93 & 7.89 & 2.49 & No apto \\
\hline 393194.27 & 18.2 & 100 & 0 & 0 & 0 & No apto \\
\hline 393194.1 & 17.9 & 49.93 & 15.8 & 10.03 & 5.59 & No apto \\
\hline 393280.57 & 13.8 & 100 & 0 & 0 & 0 & No apto \\
\hline 393280.82 & 15.9 & 100 & 0 & 0 & 0 & No apto \\
\hline 393349.68 & 19.7 & 72.03 & 11.01 & 0 & 10.96 & No apto \\
\hline 393371.58 & 16.2 & 100 & 0 & 0 & 0 & No apto \\
\hline 393371.159 & 15.3 & 100 & 0 & 0 & 0 & No apto \\
\hline 393658.44 & 18.9 & 66.42 & 0 & 0 & 3.44 & No apto \\
\hline TPS-25XTPS-67 (IP88008) & 16.40 & 57.56 & 3.45 & 3.80 & 7.18 & No apta \\
\hline MF-IIxTPS-67 (IP88004) & 15.70 & 100 & 0 & 0 & 0 & No apta \\
\hline Achiranax TS-4 (990021) & 16.20 & 88.45 & 1.18 & 0 & 8.94 & No apta \\
\hline Andinita & 16.7 & 100 & 0 & 0 & 0 & No apta \\
\hline Tibisay & 11.3 & 31.45 & 5.92 & 4.02 & 3.26 & No apta \\
\hline Cartayita & 15.1 & 23.33 & 36.67 & 21.67 & 5.67 & No apta \\
\hline Esperanza & 15.90 & 96.40 & 0.5 & 0 & 2.89 & No apta \\
\hline Caribay & 15.80 & 89.53 & 0 & 2.19 & 3.95 & No apta \\
\hline Unica Peruana & 15.80 & 100 & 0 & 0 & 0 & No apta \\
\hline SN Táchira & 16,8 & 100 & 0 & 0 & 0 & No apta \\
\hline Montañita & 20.4 & 45.63 & 3.82 & 8.7 & 6.63 & No apta \\
\hline Granola (testigo) & 18.3 & 100 & 0 & 0 & 0 & No apta \\
\hline
\end{tabular}




\section{Caracterización morfológica}

La caracterización morfológica de los materiales permite tener el conocimiento más adecuado de las accesiones que se conservan y poder utilizarlas eficientemente (Castillo et al., 2007)

Se observó variabilidad en los 35 genotipos evaluados (Tabla 3). Predominaron los tubérculos de forma oblonga, comprimido y redondo, de piel blanca crema y pulpa blanca con ojos superficiales. El carácter profundidad de ojos es importante tener en cuenta en los programas de mejoramiento, donde se trata siempre obtener variedades con ojos superficiales (Castillo et al., 2007) Estos resultados coinciden con los obtenidos por Meza et al. (2010) cuando evaluaron el comportamiento de clones en Marajabú estado Trujillo.

Tabla 3. Caracterización morfológica de genotipos de papa del estado Mérida, Venezuela.

\begin{tabular}{|c|c|c|c|c|c|}
\hline $\begin{array}{c}\text { Clones, progenies, } \\
\text { variedades }\end{array}$ & Forma tubérculo & Color piel & $\begin{array}{c}\text { Color } \\
\text { secundario } \\
\text { tubérculo }\end{array}$ & Color pulpa & $\begin{array}{c}\text { Profundidad } \\
\text { ojos }\end{array}$ \\
\hline 301002.6 & $\begin{array}{l}\text { Comprimido a } \\
\text { redondo }\end{array}$ & Amarillo pálido & $\mathrm{np}$ & Blanca & $\mathrm{S}$ \\
\hline 382151.22 & Obovado & Blanco crema intenso & np & blanca & M \\
\hline 382121.25 & Aplanada & Blanco crema intenso & np & blanca & M \\
\hline 386528.7 & $\begin{array}{l}\text { comprimida y } \\
\text { oblonga }\end{array}$ & $\begin{array}{l}\text { Blanco crema } \\
\text { intermedio }\end{array}$ & $\mathrm{np}$ & blanca & M \\
\hline 391011.17 & ovoide & Amarillo palido & $\mathrm{np}$ & crema & S \\
\hline 391580.30 & redondo & $\begin{array}{c}\text { Anaranjadopalido } \\
\text { Rugosa }\end{array}$ & $\mathrm{np}$ & blanca & $S$ \\
\hline 392633.54 & $\begin{array}{l}\text { Obovado } \\
\text { aplanado }\end{array}$ & Blanco crema & $\mathrm{np}$ & crema & $\mathrm{S}$ \\
\hline 392639.1 & Comprimida & Blanco crema intenso & $\mathrm{np}$ & blanca & $\mathrm{P}$ \\
\hline 393085.5 & Oblongo & blanco crema & $\mathrm{np}$ & crema & $S$ \\
\hline 393194.27 & comprimida & blanco crema pálido & $\mathrm{np}$ & blanca & $\mathrm{P}$ \\
\hline 393194.1 & comprimido & blanco crema pálido & np & blanca & $\mathrm{P}$ \\
\hline 393280.57 & obovado & $\begin{array}{l}\text { Blanco crema } \\
\text { intermedio }\end{array}$ & $\begin{array}{l}\text { Rosado } \\
\text { jaspeado }\end{array}$ & crema & M \\
\hline 393280.82 & obovado & Blanco crema & $\begin{array}{c}\text { Rosado, ojos } \\
\text { pigmentados } \\
\text { y jaspeado }\end{array}$ & crema & M \\
\hline 393349.68 & $\begin{array}{l}\text { Oblongo } \\
\text { aplanado }\end{array}$ & Blanco crema & $\begin{array}{l}\text { con color sec. } \\
\text { rosado en las } \\
\text { cejas }\end{array}$ & amarilla & $S$ \\
\hline
\end{tabular}


Continuación... Tabla 3

\begin{tabular}{|c|c|c|c|c|c|}
\hline $\begin{array}{l}393371.58 \\
\text { (Chucmarina) }\end{array}$ & Oblongo & Blanco crema & $\begin{array}{c}\text { con rosado en } \\
\text { cejas }\end{array}$ & crema & $\mathrm{S}$ \\
\hline 393371.159 & obovado & Blanco crema & $\begin{array}{l}\text { Rosado en } \\
\text { ojos }\end{array}$ & blanca & $\mathrm{S}$ \\
\hline 393658.44 & comprimida & blanco crema pálido & np & blanca & $\mathrm{P}$ \\
\hline $\begin{array}{l}\text { TPS-25 XTPS-13 } \\
\text { (IP88007) } \\
\text { Granate INIA }\end{array}$ & $\begin{array}{l}\text { Comprimido a } \\
\text { redondo }\end{array}$ & Marrón pálido & np & crema & M \\
\hline $\begin{array}{l}\text { TPS-25XTPS-67 } \\
\text { (IP88008) }\end{array}$ & $\begin{array}{l}\text { Redondos y } \\
\text { oblongos }\end{array}$ & Blanco crema & np & crema & M \\
\hline $\begin{array}{l}\text { MF-IIxTPS-67 } \\
\text { (IP88004) }\end{array}$ & Redondos & Marrón pálido & np & crema & M \\
\hline $\begin{array}{l}\text { AchiranaxTPS-67 } \\
\text { (96003) }\end{array}$ & Oblongos & Marrón pálido & $\mathrm{np}$ & crema & M \\
\hline $\begin{array}{l}\text { Achiranax } \\
\text { (990021) }\end{array}$ & $\begin{array}{l}\text { Oblongos y } \\
\text { redondos }\end{array}$ & Marrón pálido & $\mathrm{np}$ & crema & M \\
\hline Andinita & oblonga & Blanco crema & $\mathrm{np}$ & blanca & $\mathrm{S}$ \\
\hline Tibisay & redondo & Blanco crema & $\mathrm{np}$ & crema & $\mathrm{S}$ \\
\hline Cartayita & Redondo & Marrón pálido & $\mathrm{np}$ & crema & $\mathrm{S}$ \\
\hline Esperanza & Redonda & Marrón pálido & $\begin{array}{c}\text { rojo morado } \\
\text { cejas, ojos y } \\
\text { manchas } \\
\text { dispersas }\end{array}$ & crema & $S$ \\
\hline Iniafrit & Oblongo alargado & Blanco crema & $\mathrm{np}$ & crema & $S$ \\
\hline Caribay & Redondos & Crema & $\begin{array}{l}\text { con ligero } \\
\text { tono rosado }\end{array}$ & Blanco crema & M \\
\hline Fripapa INIA & Oblongo & Rosado intenso & np & amarilla & M \\
\hline Unica Peruana & Oblongo & Rojo & np & crema & $S$ \\
\hline Diacol Capiro & $\begin{array}{l}\text { Redondo } \\
\text { ligeramente } \\
\text { aplanado }\end{array}$ & Rojo morado & $\mathrm{np}$ & crema & $S$ \\
\hline Maria Bonita & oblongo & Blanco crema & np & crema & $S$ \\
\hline SN Táchira & Comprimida & Blaco crema intenso & $\mathrm{np}$ & crema & M \\
\hline Montañita & Oblonga & $\begin{array}{l}\text { Blanco crema } \\
\text { intermedio }\end{array}$ & np & blanca & M \\
\hline Granola (testigo) & Oval redondeada & Amarilla clara & $\mathrm{np}$ & Amarilla clara & \\
\hline
\end{tabular}

$\mathrm{np}=$ no presentó, $\mathrm{S}=$ superficiales, $\mathrm{M}=$ medios, $\mathrm{P}=$ profundos 


\section{Agradecimientos}

Los autores agradecen al Centro Internacional de la Papa por los genotipos aportados y apoyo técnico - científico en el desarrollo de este trabajo. A la Red Latinpapa por el aporte económico junto al Instituto de Investigaciones Agropecuarias de Venezuela (INIA), a los agricultores que participaron en las evaluaciones $\mathrm{y}$ a todo el personal de apoyo.

\section{Conflictos de intereses}

El documento no tiene conflicto de intereses con las instituciones donde fueron realizadas las investigaciones.

\section{Referencias citadas}

Bonierbale, M.; de Haan S.; Forbes, A. (2010) Procedimientos para pruebas de evaluación estándar de clones avanzados de papa. Centro Internacional de la Papa, Lima, Perú. 124 p.

Castillo, J.; Estévez, A.; Salomón, J; Pérez, A.; Ortiz, U. (2007). Caracterización morfoagronómica del germoplasma cubano de papa (Solanum ssp.). Evaluación de las especies silvestres. Parte I. Cultivos Tropicales. 28(1):63-68.

Forbes, G.; Pérez, W.; Andrade Piedra, J. (2014). Procedimiento para Evaluación Estándar y Manejo de Datos de Clones Avanzados de Papa. Modulo 3: Evaluación de la resistencia en genotipos de papa a Phytophthora infestans bajo condiciones de campo. Guía para Colaboradores Internacionales. Lima (Perú). Centro Internacional de la Papa (CIP). $50 \mathrm{p}$.

Gabriel, J.; Castro, C.; Valverde, A.; Indacochea, B. (2017). Diseños experimentales experimentos agropecuarios. Grupo Compas, Universidad Estatal del Sur de Manabí, Jipijapa, Manabí, Ecuador.

González. L.; Niño L.; Gastelo, M.; Suárez, F. (2011). Evaluación y selección de clones de papa con resistencia a candelilla tardía en el estado Mérida, Venezuela. Revista Latinoamericana de la papa 16 (1) 142-150.

Madroñero, I. C.; Rosero, J.E.; Rodríguez, L.E.; Navia, J.F.; Benavides, C. A. (2013). Caracterización Morfoagronómica de genotipos promisorios de papa criolla (Solanum tuberosum L. Grupo Andigenum) en Nariño. Temas Agrarios vol.18(2): 50-66.

Huamán, Z.; Gómez, R. (1994). Descriptores Morfológicos de la Papa para la caracterización básica de colecciones nacionales. Ed. del Centro Internacional de la papa, Lima, Perú. 65 p.

Martínez, M. N. y Ligareto, G. A. (2005). Evaluación de cinco genotipos promisorios de papa Solanum tuberosum sp. Andígena según desempeño agronómico y calidad industrial. Agronomía Colombiana 23(1):17-27.

Meza, N.; Herrera, J.; Gudiño, S. (2009). Comportamiento de clones promisorios de papa (Solanum tuberosum L.) en la localidad de Cuencas, estado Trujillo, Venezuela. Bioagro 21 (2): 149-151

Meza, N., Herrera, J.; López, J. (2010). Comportamiento de clones de papa en las condiciones de Marajabú, estado Trujillo, Venezuela. INIA Divulga enero-abril $2010(43-45)$

Monar, C.; Silva, D.; Velasco, I.; Guambuguete, I. (2015). Evaluación Agronómica de cuatro clones promisorios y tres variedades de papa (Solanum 
tuberosum L.) con investigación participativa, en cuatro localidades de la provincia Bolívar. Revista de Investigación Talentos vol. I n ${ }^{\circ}$ 1:77-83.

Niño, L.; González, L.; Villamizar, E.; Acevedo, E.; Becerra, F. (2004). Evaluación de Variedades y Clones Avanzados de Papa (Solanum tuberosum L.) con Características para el Procesamiento Industrial en el Estado Mérida, Venezuela. Revista Fitotecnia Colombiana 4 (1): 1-8.

Poehlman, J.M. (2003). Mejoramiento Genético de las Cosechas. Aplicaciones en cultivos extensivos que se propagan vegetativamente. Capitulo 21 Mejoramiento genético de la papa. Editorial Limusa. Páginas 435-449. México

Rodríguez, D.; Alcalá, D.; Escalona, F. (2008). Selección de clones de papa por resistencia a la candelilla tardía y rendimiento. Bioagro 20 (1): 29-35.

Rodríguez D., M. Ojeda, M. Pérez de Camacaro, M. Gallardo, R. Valera y F. Bittara. (2009). Producción, incidencia de la sarna polvorienta y calidad de clones avanzados de papa. Rev. Fac. Agron. 26 (4):508-531. 